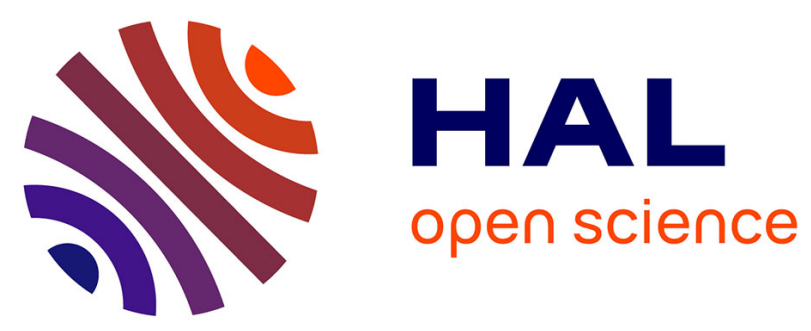

\title{
Orientation de la réponse immune par les basophiles
}

\author{
C. Galeotti, E. Stephen-Victor, M. Sharma, S.V. Kaveri, J. Bayry
}

\section{To cite this version:}

C. Galeotti, E. Stephen-Victor, M. Sharma, S.V. Kaveri, J. Bayry. Orientation de la réponse immune par les basophiles. Revue francaise d'allergologie, 2016, 10.1016/j.reval.2016.01.025 . hal-01285509

\section{HAL Id: hal-01285509 \\ https://hal.sorbonne-universite.fr/hal-01285509}

Submitted on 9 Mar 2016

HAL is a multi-disciplinary open access archive for the deposit and dissemination of scientific research documents, whether they are published or not. The documents may come from teaching and research institutions in France or abroad, or from public or private research centers.
L'archive ouverte pluridisciplinaire HAL, est destinée au dépôt et à la diffusion de documents scientifiques de niveau recherche, publiés ou non, émanant des établissements d'enseignement et de recherche français ou étrangers, des laboratoires publics ou privés. 


\section{Orientation de la réponse immune par les basophiles \\ Orientation of the immune response by basophils}

Caroline Galeotti ${ }^{1,2,3,4}$ Emmanuel Stephen-Victor ${ }^{1,2,3}$, Meenu Sharma ${ }^{1,3}$, Srini V Kaveri $^{1,2,3,5}$, Jagadeesh Bayry ${ }^{1,2,3,5, *}$

${ }^{1}$ Institut National de la Santé et de la Recherche Médicale Unité 1138, Paris, F75006, France

${ }^{2}$ Sorbonne Universités, UPMC Univ Paris 06, UMR S 1138, Paris, F-75006, France ${ }^{3}$ Centre de Recherche des Cordeliers, Equipe - Immunopathologie et immunointervention thérapeutique, Paris, F-75006, France

${ }^{4}$ Department of pediatric rheumatology, National referral centre of auto-inflammatory diseases, CHU de Bicêtre, 94270 Le Kremlin-Bicêtre, France

${ }^{5}$ Université Paris Descartes, Sorbonne Paris Cité, UMR S 1138, Paris, F-75006, France

Auteur correspondant: Jagadeesh Bayry, Institut National de la Santé et de la Recherche Médicale Unité 1138, Centre de Recherche des Cordeliers, 15 rue de l'Ecole de Médicine, Paris, F-75006, France. Tel: 0033144278203 ; Fax: 00331 44278194

E-mail: jagadeesh.bayry@crc.jussieu.fr

Mots clé : basophiles ; cellule présentatrice d'antigène; IL-4 ; réponse Th2 ; Th17 Key words : basophils; antigen presenting cells; IL-4; Th2 response; Th17 


\section{Résumé}

Les basophiles sont de rares granulocytes et ont un rôle important dans les réponses Th2, dans la protection contre les helminthes et dans la pathogénèse de l'allergie et d'autres maladies inflammatoires. Des études récentes ont suggéré que les basophiles fonctionnent comme des cellules présentatrices d'antigènes pour médier les réponses Th2 chez la souris. Cependant, les basophiles humains n'ont pas les caractéristiques des cellules présentatrices d'antigène.

\section{Abstract:}

Basophils are rare granulocytes and are important for Th2 responses, protection against helminth parasites and pathogenesis of allergy and other inflammatory conditions. Recent reports suggest that in mice basophils function as antigen presenting cells for mediation of Th2 responses. However, human basophils lack features of antigen presenting cells.

\section{Introduction}

Les basophiles sont les granulocytes sanguins les plus rares. Ils représentent moins de $1 \%$ des leucocytes circulants. Leur rôle important dans la protection contre les helminthes et dans la pathogénèse des maladies allergiques est bien connu. Les marqueurs de surface permettant de les identifier et de les isoler incluent RFcEl, CD203c et CD123. Les basophiles expriment également plusieurs marqueurs d'activation qui permettent de suivre leur état, notamment CD69, CD63, CD164, CD13, CD107a et CD11b. Comme ils expriment un grand nombre de récepteurs capables d'induire leur activation, dont les TLR 2 et 4, les récepteurs de l'IL-3, I'IL-33 et l'IL-25 et les RFcEl ayant une haute affinité pour les lgE, les basophiles peuvent répondre rapidement à différents stimuli et sécréter des immnunomodulateurs comme des cytokines (IL4, IL-6, IL-33, TSLP, BAFF), des chemokines, de l'histamine, des protéoglycanes et des leucotriènes.

Les basophiles activés contribuent à la polarisation d'une réponse Th2 et augmentent les réponses Th2 médiées par les cellules dendritiques (CDs). Récemment, il a été montré que la fonction des basophiles dans la polarisation des 
réponses Th2 est non seulement importante pour la protection contre les helminthes, mais également dans certaines pathologies comme l'asthme, l'allergie et les maladies autoimmunes comme le lupus érythémateux disséminé [1].

\section{Basophiles et réponse Th2}

Le rôle des basophiles dans la polarisation des réponses Th2 via la sécrétion de l'IL4 est connue depuis longtemps. Les basophiles sont activés et recrutés au niveau des ganglions lymphatiques drainants spécifiquement en réponse à un allergène induisant une réponse Th2. De plus, il a été démontré que le basophile est le type de cellule accessoire requis pour l'induction d'une réponse Th2 en réponse à des allergènes de type protéase. Les basophiles sont directement activés par des allergènes de type protéase et produisent des cytokines induisant une réponse Th2, incluant l'IL-4 et le TSLP, qui sont impliquées dans la différentiation Th2 in vivo [2] (Figure 1).

Le rôle non redondant des basophiles dans l'établissement d'une réponse Th2 a été démontré en les déplétant dans différents modèles murins d'infection par des helminthes et des tiques. Le recrutement des basophiles sur le site d'infestation des tiques au cours d'une deuxième infestation permet de réduire le temps de contact avec les tiques, et donc le risque d'infection. Cela révèle qu'ils sont le support effecteur d'une mémoire immunologique contre ces infestations [3]. La protection développée contre les helminthes Nippostrongylus brasiliensis ou Heligmosomoides polygyrus est dépendante de la sécrétion d'IL-4 et/ou d'IL-33 par les basophiles, de leur expression de la chaine commune $y$ des récepteurs RFc et de la présence d'IgE [4].

Les basophiles augmentent aussi la réponse humorale mémoire en augmentant la prolifération cellulaire $B$ et la production d'anticorps. Ces fonctions des basophiles sont induites par I'IL-6 et I'IL-4 et par l'augmentation de la capacité des cellules T $\mathrm{CD} 4^{+}$à fournir de l'aide aux cellules $\mathrm{B}$ [5].

\section{Les basophiles sont des cellules présentatrices d'antigène chez la souris, mais pas chez l'homme}

Des rapports récents chez la souris ont montré que les basophiles fonctionnent comme des cellules présentatrices d'antigènes (CPA). Ils expriment le $\mathrm{CMH}$ de classe II et des molécules de co-stimulation CD80 et CD86, capture et présente les 
antigènes solubles, les antigènes de type protéase ou les complexes lgE-antigènes et polarisent la réponse Th2 [6]. Cependant, le rôle des basophiles comme CPA dans la polarisation des réponses Th2 in vivo est soumis à controverse à cause des méthodes de déplétion utilisées dans les expériences.

Mais contrairement aux basophiles murins, les basophiles humains n'expriment pas le HLA-DR et les molécules de co-stimulation CD80 et CD86 [7] ni à l'état basal ni lorsqu'ils sont activés par des antigènes. En effet, lorsque les basophiles sont stimulés avec des allergènes communs tels que Asp f 1 et Bet $v 1$, ils n'expriment pas non plus le HLA-DR et les molécules de co-stimulation CD80 et CD86. De plus, la co-culture de CDs pulsées avec Asp f 1 et de cellules T CD4+ permet de promouvoir une réponse Th2 analysée par la sécrétion d'IL-4. Cependant, les basophiles pulsés avec Asp f 1 n'ont pas réussi à induire une réponse cellulaire $T$ quand ils sont en co-culture avec les cellules T CD4+ [7]. Ces données suggèrent que les basophiles humains ne sont pas capables de promouvoir des réponses Th2 comme CPA.

\section{Les basophiles humains n'ont pas la capacité de promouvoir une réponse Th17}

Une population de lymphocytes T CD4+ récemment décrite, les cellules Th17, est également impliquée dans la pathogenèse de l'asthme, de l'allergie chronique, du lupus et d'autres maladies auto-immunes et inflammatoires, suggérant que les basophiles pourraient induire leur activation et leur expansion.

Notre équipe a cependant démontré que les basophiles activés par l'IL-3 et/ou le récepteur RFcEl n'étaient pas capables d'expandre les Th17, ni d'induire la production d'IL-17 directement ou indirectement par les CPA comme les monocytes [8]. Comme les basophiles humains n'ont pas d'HLA-DR et de molécules de costimulation, leur incapacité à transmettre des signaux médiés par les récepteurs des cellules $T$ et les molécules de co-stimulation aux lymphocytes $T$ CD4+ pourrait expliquer l'absence de réponse Th17. II a également été montré que les lymphocytes T CD4+ ne sécrètent pas d'IL-22 en présence de basophiles activés [9].

L'absence de capacité des basophiles humains à promouvoir une réponse Th17 a également été démontrée dans un autre système utilisant des antigènes de Schistosoma mansoni. Comme les basophiles ont un rôle important dans la 
régulation de la réponse immunitaire face aux helminthes comme $S$. mansoni, nous avons exploré si les réponses Th17 observées dans la schistosomiase humaine étaient médiées par les basophiles. Nous avons montré que les réponses Th17 sont médiées par les CD et non par les basophiles [10].

\section{Conclusions}

L'IL-4 sécrétée par les basophiles joue un rôle majeur dans la promotion des réponses Th2 médiées par des CPA comme les CDs. Cependant, les CDs sont capables de polariser les réponses Th2 indépendamment de l'IL-4, par exemple grâce à ligand OX-40L [11]. Chez la souris, les basophiles expriment le $\mathrm{CMH}$ de classe II et les molécules de co-stimulation et présentent des antigènes solubles, des allergènes, des haptènes et des peptides pour la différentiation Th2. En revanche, les basophiles humains circulants ne présentent pas les caractéristiques des CPA.

\section{Déclaration d'intérêts}

Les auteurs déclarent ne pas avoir de conflits d'intérêts en relation avec cet article.

\section{Remerciements}

Ce travail a été réalisé avec le soutien financier de l'Inserm, de l'Université Pierre et Marie Curie, de I'Université Paris Descartes, de la Fondation pour la Recherche Médicale (bourse de la FRM FDM20150633674 à C. Galeotti) et de la Centre Franco-Indien pour la Promotion de la Recherche Avancée (bourse de la CEFIPRA 4803-1 à E. Stephen-Victor). 


\section{Références}

1. Sharma M, Bayry J. Autoimmunity: Basophils in autoimmune and inflammatory diseases. Nat Rev Rheumatol 2015,11:129-131.

2. Sokol CL, Barton GM, Farr AG, Medzhitov R. A mechanism for the initiation of allergen-induced T helper type 2 responses. Nat Immunol 2008,9:310-318.

3. Wada $T$, Ishiwata $\mathrm{K}$, Koseki $\mathrm{H}$, Ishikura $\mathrm{T}$, Ugajin $\mathrm{T}$, Ohnuma $\mathrm{N}$, et al. Selective ablation of basophils in mice reveals their nonredundant role in acquired immunity against ticks. J Clin Invest 2010,120:2867-2875.

4. Schwartz C, Turqueti-Neves A, Hartmann S, Yu P, Nimmerjahn F, Voehringer D. Basophil-mediated protection against gastrointestinal helminths requires IgE-induced cytokine secretion. Proc Natl Acad Sci U S A 2014,111:E51695177.

5. Denzel A, Maus UA, Rodriguez Gomez M, Moll C, Niedermeier M, Winter C, et al. Basophils enhance immunological memory responses. Nat Immunol 2008,9:733-742.

6. Perrigoue JG, Saenz SA, Siracusa MC, Allenspach EJ, Taylor BC, Giacomin $\mathrm{PR}$, et al. MHC class II-dependent basophil-CD4+ T cell interactions promote $\mathrm{T}(\mathrm{H}) 2$ cytokine-dependent immunity. Nature Immunol 2009,10:697-705.

7. Sharma M, Hegde P, Aimanianda V, Beau R, Maddur MS, Senechal H, et al. Circulating human basophils lack the features of professional antigen presenting cells. Sci Rep 2013,3:1188.

8. Sharma M, Stephen-Victor E, Poncet P, Kaveri SV, Bayry J. Basophils are inept at promoting human Th17 responses. Hum Immunol 2015,76:176-180.

9. Sharma M, Kaveri SV, Bayry J. Human basophils lack the capacity to drive memory CD4(+) T cells toward the IL-22 response. J Allergy Clin Immunol 2013,132:1457-1458.

10. Sharma M, Lecerf M, Friboulet A, Kaveri SV, Dissous C, Bayry J. Mediation of T-helper 17 responses to schistosomes by dendritic cells but not basophils. J Infect Dis 2014,209:2019-2021.

11. Maddur MS, Sharma M, Hegde P, Stephen-Victor E, Pulendran B, Kaveri SV, et al. Human B cells induce dendritic cell maturation and favour Th2 polarization by inducing OX-40 ligand. Nature Commun 2014,5:4092. 


\section{Légende de la figure}

Figure 1. Orientation de la réponse immune par les basophiles. Des cytokines comme l'IL-3, l'IL-33 et le TSLP, la stimulation des TLR 2 et 4, et des complexes immuns avec des lgE activent les basophiles. Ces basophiles activés produisent des cytokines comme l'IL-4 et le TSLP, qui vont promouvoir les réponses Th2. Les cellules Th2 ainsi que les basophiles (via l'IL-4, l'IL-6 et BAFF) induisent l'activation, la différentiation des cellules $B$ et la production d'anticorps (incluant les lgE).

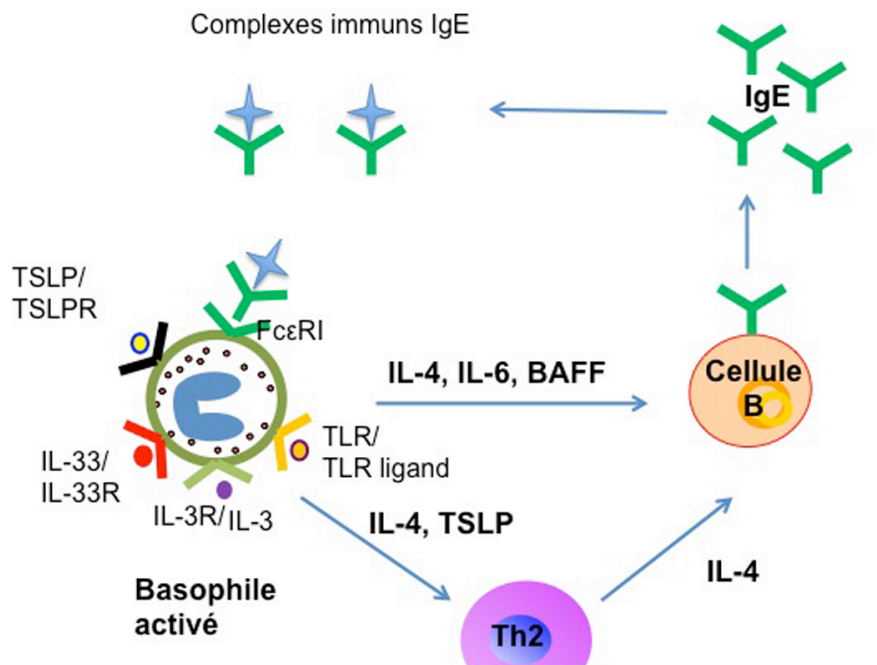

